Supporting Information

\title{
Lake Huron's Phosphorus Contributions to the St. Clair/Detroit River Great Lakes Connecting Channel
}

\author{
Donald Scavia $^{a}$, Eric J. Anderson ${ }^{b}$, Alice Dove ${ }^{c}$, Brad Hill', Colleen M. Longd, Yu-Chen \\ Wang $^{\mathbf{a}}$
}

a, ${ }^{*}$ School for Environment and Sustainability, University of Michigan, 440 Church St. Ann Arbor, Michigan 48109, United States

${ }^{\mathrm{b}}$ Great Lakes Environmental Research Laboratory, National Oceanic and Atmospheric Administration, 4840 S. State Rd., Ann Arbor, Michigan, 48108, United States

${ }^{\mathrm{c}}$ Water Quality Monitoring \& Surveillance Division, Environment and Climate Change Canada, 867 Lakeshore Rd., Burlington, Ontario L7S 1A1 Canada

${ }^{\mathrm{d} B a r r}$ Engineering, 4771 50th St. Grand Rapids, Michigan, 49512, United States

7 pages with 7 figures 
Estimating depth at which waves influence the bottom. As a wind wave moves inland from deep water, its speed and length are first only a function of its period (or frequency). As water depth becomes shallower relative to the wave's length, the length and speed are dependent upon both the depth and period. The boundary between deep and transitional waves (i.e., when the bottom begins to influence the wave, is when water depth (d) divided by the wavelength (L) is greater than 0.5. This is therefore where the waves start to impact the bottom, and resuspension is possible. That depth can be estimated, based on linear wave theory, as $\mathrm{gT}^{2} / 4 \pi$, where $\mathrm{g}$ is the acceleration due to gravity (9.81) and $\mathrm{T}$ is the mean wave period (s) (USACE 2003). We assumed that waves with these characteristics have the potential to resuspend sediment from that point shoreward.

We used hindcast wave periods reported in the Corps of Engineers Wave Information Studies project (WIS 2019). Unlike forecasts, wave hindcasts predict past wave conditions using models and observed wind fields. By using wind fields, hindcast wave information is generally of higher accuracy than forecasts, is often representative of observed wave conditions, and can be provided for locations where wave heights were not measured.

To generate wave characteristics based on direct observations, one would conduct a spectral wave analysis based on lake-surface time-series typically over periods from 17 to 68 minutes, and measured at roughly one sample per second $(1 \mathrm{~Hz})$. There are two main types of sensors used to measure surface elevation, pressure sensors and buoys. Pressure sensors are mounted at a fixed position underwater, and they measure the height of the water column that passes above them. In contrast, buoys ride atop the surface. Equipped with accelerometers, the buoys can be used to calculate vertical displacements.

\section{References}

USACE 2003. Coastal Engineering Manual. Volume II. United States Army Corps of Engineers Washington, DC Manual no. 1110-2-1100.

WIS 2019. USACE Wave Information Study. http://wis.usace.army.mil/, retrieved on 12-7-2019 for Station 93266; Latitude: 43.16, Longitude: -82.16, Depth: $59 \mathrm{~m}$ and Station 93257; Latitude: 43.36, Longitude: -81.8, Depth: $66 \mathrm{~m}$. 

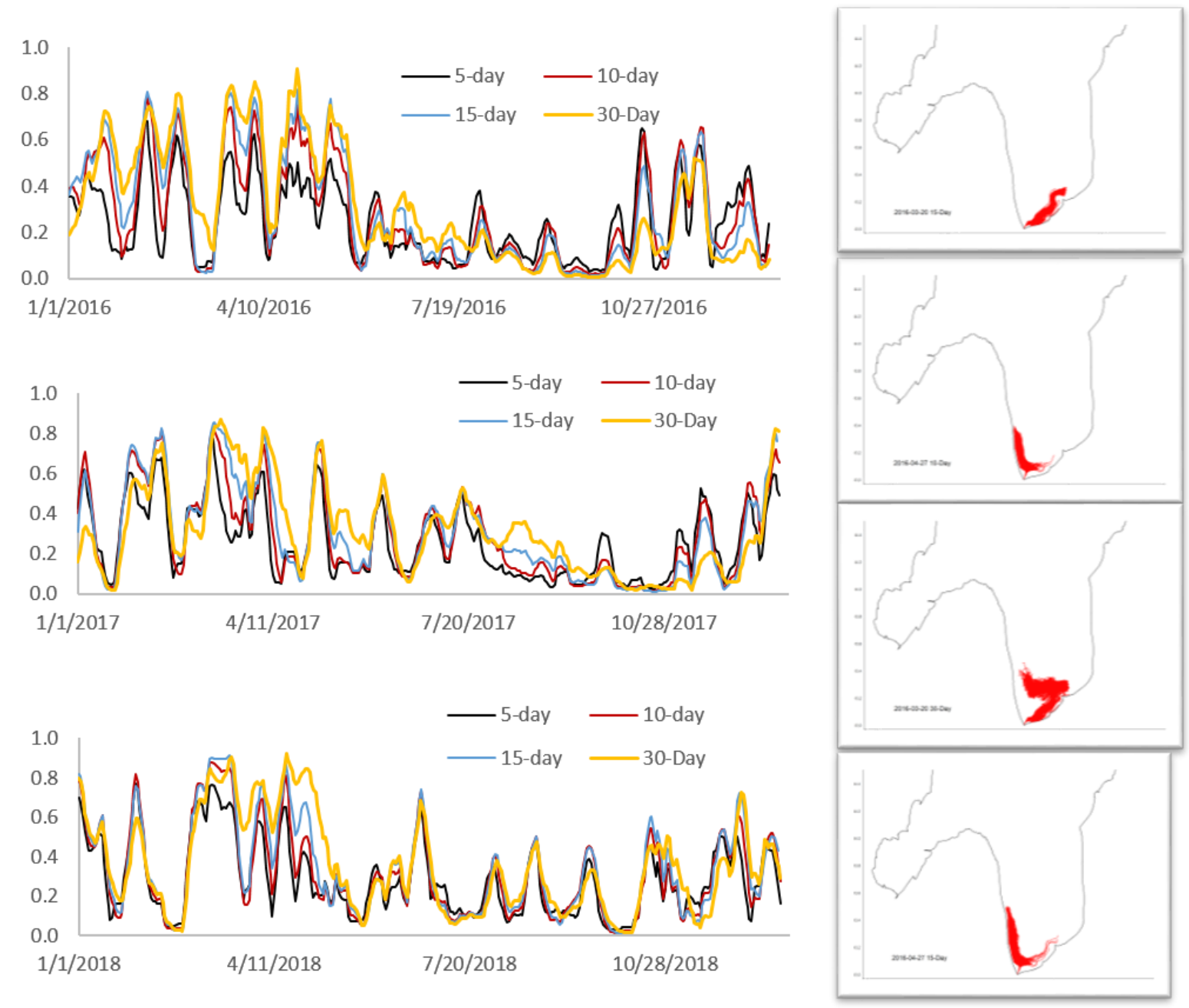

Figure S1. Left: Comparison of 5-, 10-, 15-, and 30-day percent traces for Michigan flow. Right: Examples of 15-day traces 

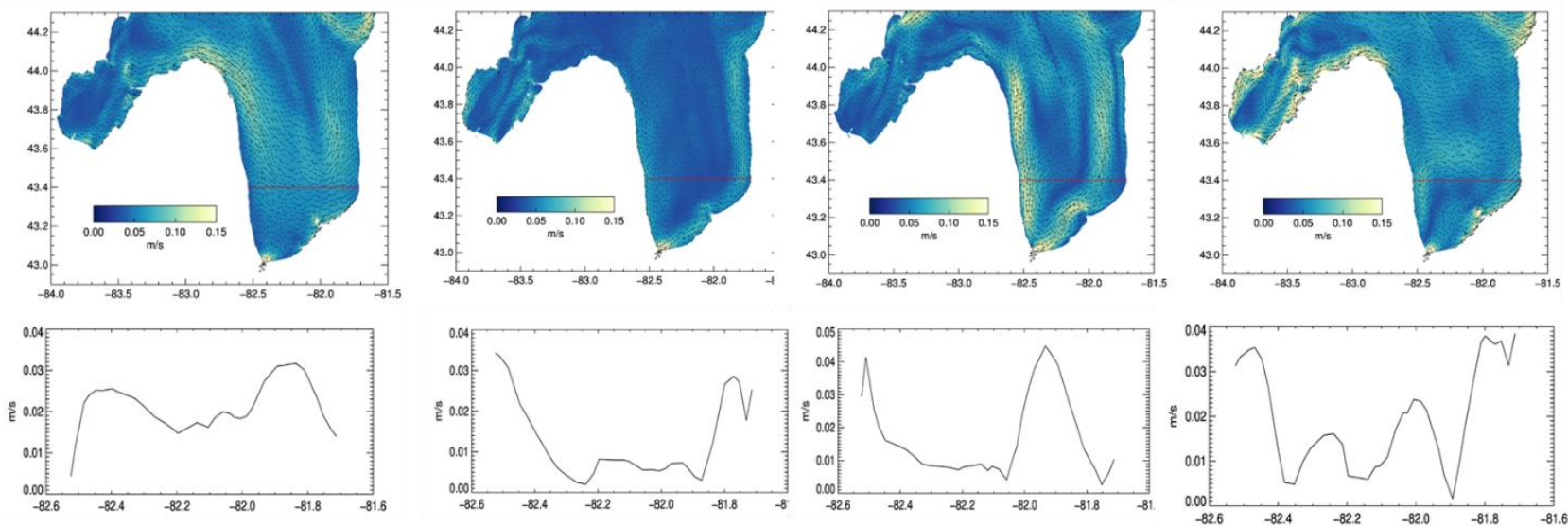

Jan-March

April-June

July-Sept

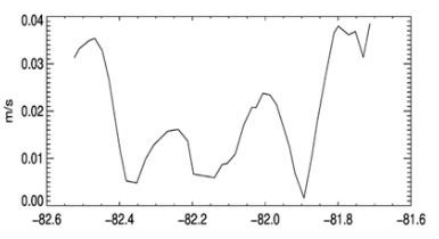

Oct-Dec

Figure S2. Top: Three-month averaged, depth-integrated currents for four 3-month periods. Bottom: Average current speeds $(\mathrm{m} / \mathrm{s})$ for the cross-sections indicated above in red.

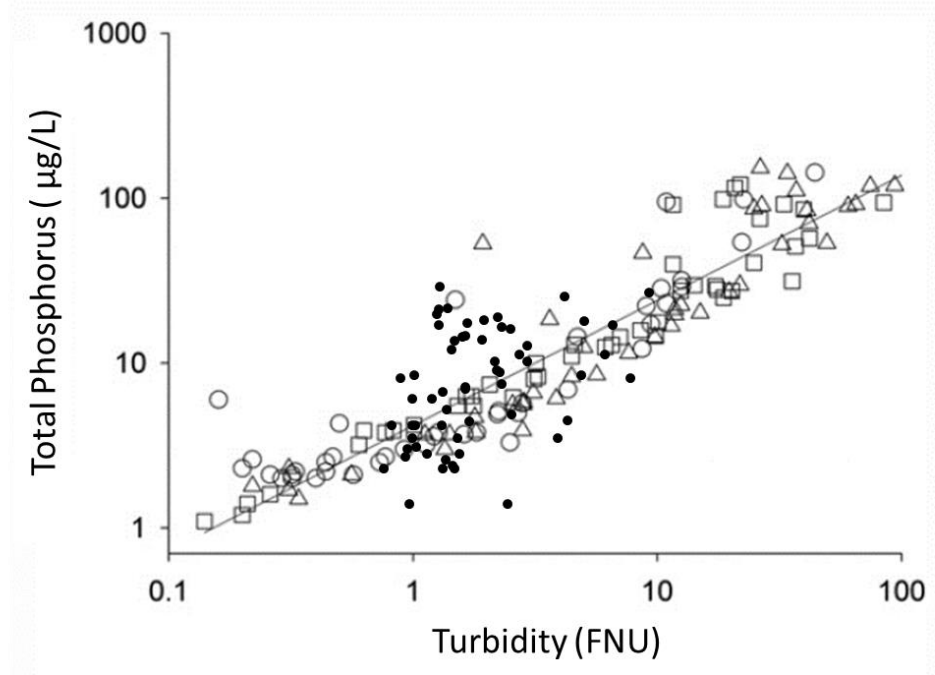

Figure S3. Relationship between total phosphorus and turbidity. Open symbols are redrawn from Howell et al. (2014) for their Point Clark study area at the north end of our study area. Black symbols are from this study. 


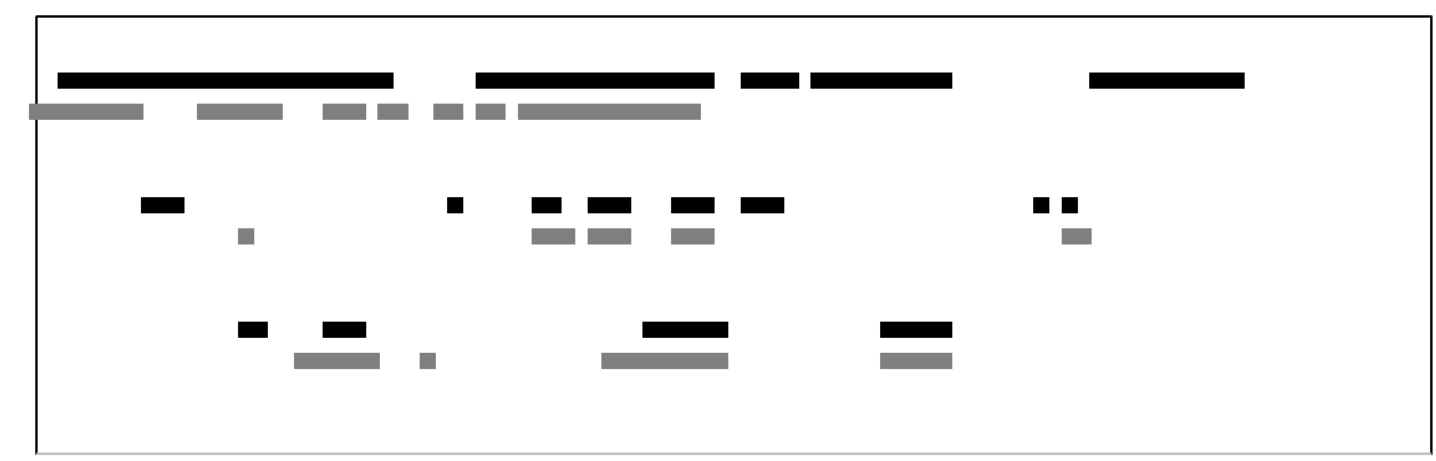

1-Jan 11-Jan 21-Jan 31-Jan 10-Feb 20-Feb 1-Mar 11-Mar 21-Mar 31-Mar 10-Apr

Figure S4. Periods of time with more than $40 \%$ ice cover for Ontario (black) and Michigan (grey) for 2016 (bottom), 2017 (middle), and 2018 (top)

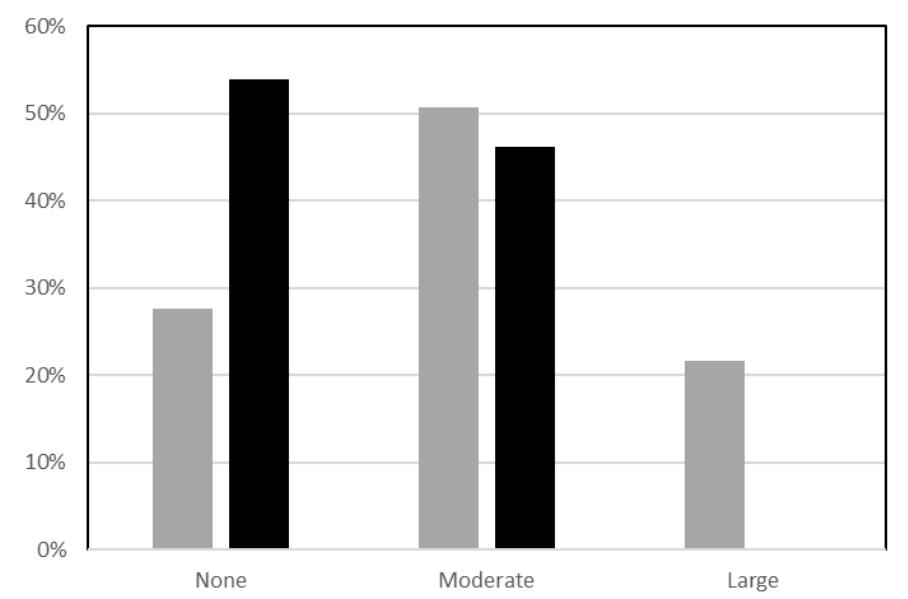

Figure S5. Frequency of large, moderate, and no resuspension in the Michigan (black) and Ontario (grey) nearshore in 2016. 

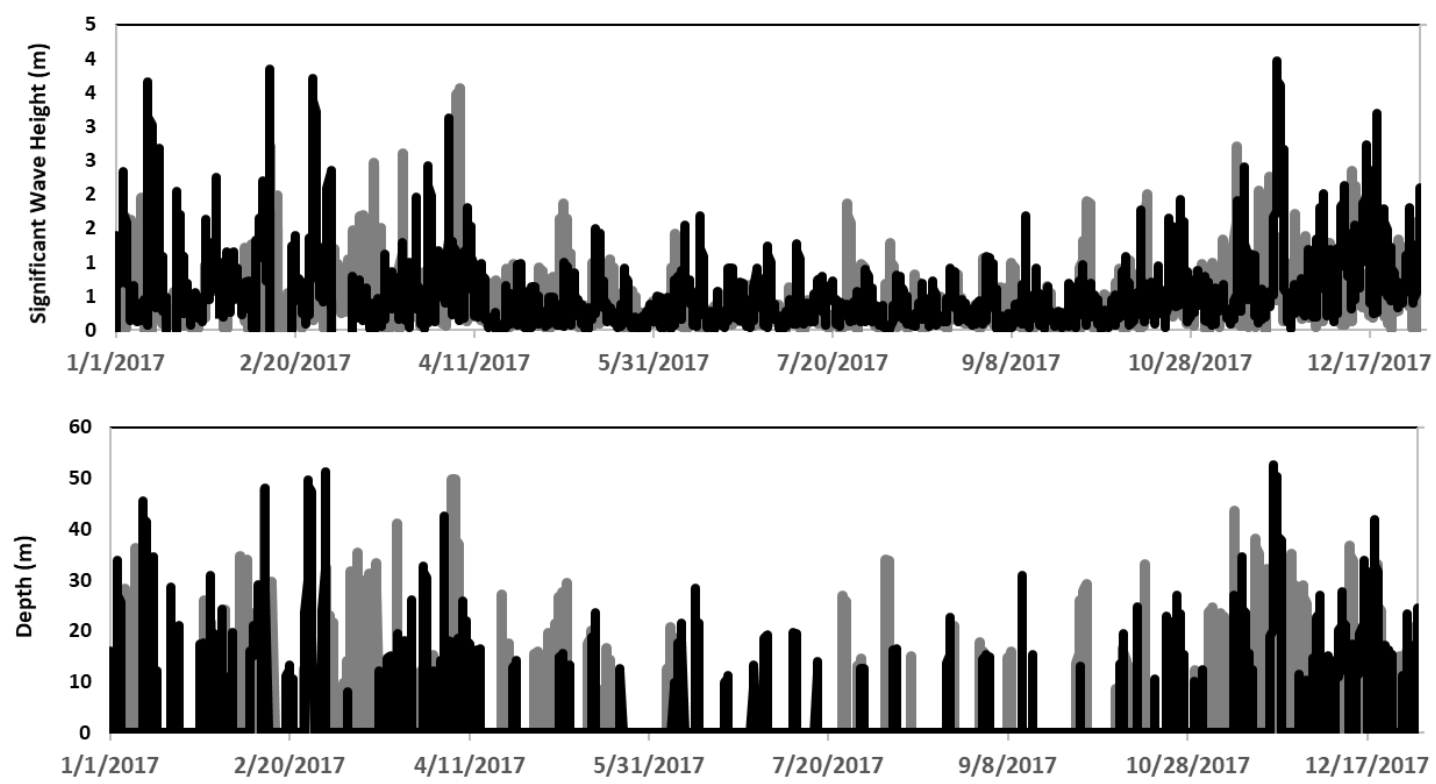

Figure S6. Significant wave heights (top) and estimated depth of bottom influence (bottom) for 2017 for station 93288 (grey) and 93257 (black)

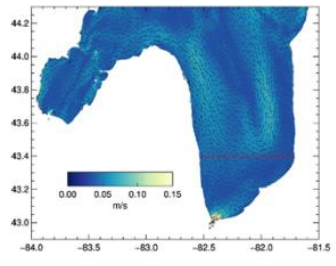

March

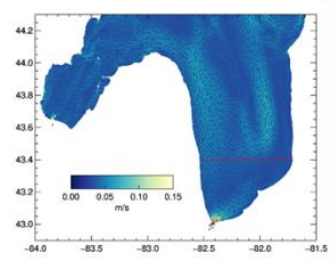

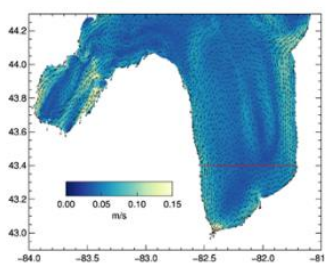

April

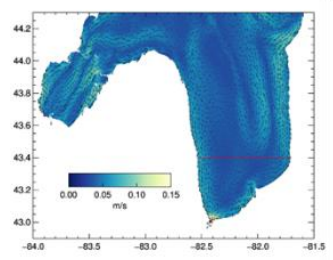

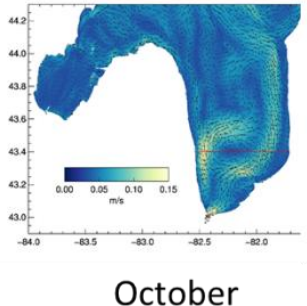

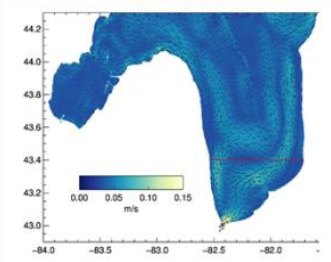

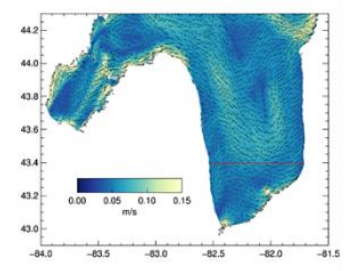

November

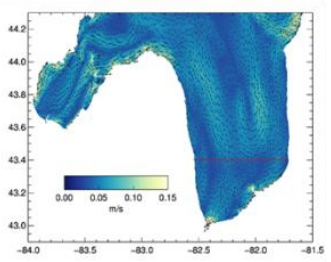

Figure S7. Monthly averaged 2016 surface (top) and depth-integrated (bottom) circulation patterns 


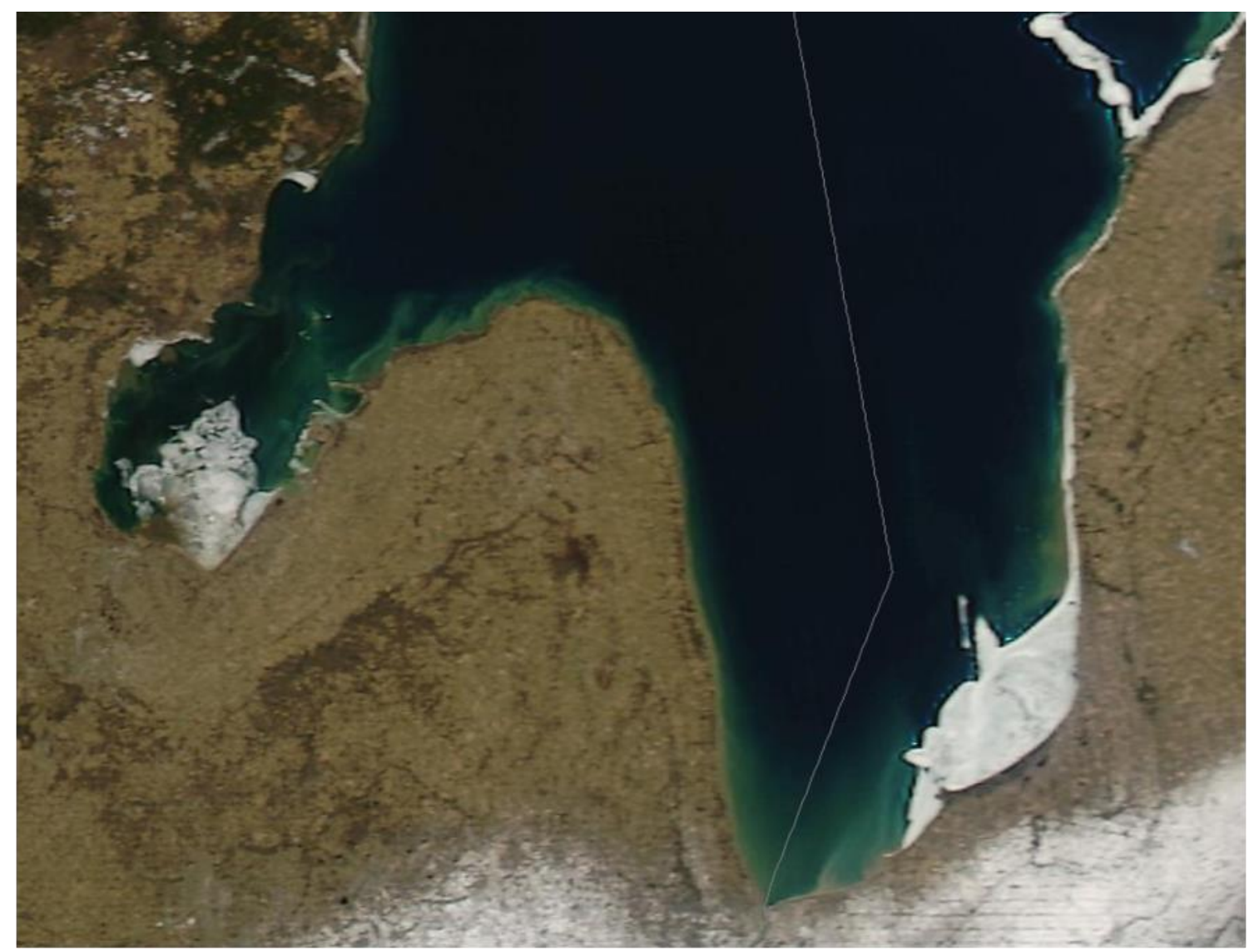

Figure S8. Modis image from March 3, 2018 showing regions of both ice cover (white) and resuspended sediment (green) along the Ontario shoreline. 